\title{
Artículo de Revisión \\ Complicaciones y manejo de procedimientos dermatoestéticos: Una actualización para el dermatólogo
}

Patricia Apt ${ }^{1}$, Javier Fernández ${ }^{2}$, María Luisa Pérez-Cotapos ${ }^{1}$

\section{RESUMEN}

En las últimas décadas se han desarrollado nuevas técnicas e indicaciones de los procedimientos estéticos mínimamente invasivos, presentando cada vez mejores resultados con un alto nivel de seguridad, sin embargo, estos no se encuentran exentos de complicaciones, las que pueden ser transitorias o permanentes. El conocimiento, abordaje, tratamiento y prevención de las distintas complicaciones son esenciales para los dermatólogos y cirujanos plásticos. Los procedimientos más utilizados son: administración de rellenos, peeling químicos, láser, luz pulsada intensa y ultrasonido de alta frecuencia. La siguiente revisión tiene como objetivo reconocer las principales complicaciones de estos procedimientos y su manejo.

Palabras clave: Complicaciones; Laser; Rellenos; Peeling químicos; Luz pulsada intensa; Ultrasonido de alta frecuencia; Tatuajes.

\section{SUMMARY}

In recent decades, new techniques and indications of minimally invasive

aesthetic procedures have been developed, presenting increasingly better

results with a high level of safety, however these are not exempt from complications, which may be transient or permanent. The knowledge, approach, treatment and prevention of the different complications are essential for the dermatologist and plastic surgeons. The most used procedures are the administration of fillers, chemical peels, lasers, intense pulsed light and high frequency ultrasound. The following review aims to recognize the main complications of these procedures and their management.

Key words: Complications; Lasers; Filler; Chemical peeling; Intense pulsed light; Microfocused ultrasound; Tattoo.
L os procedimientos estéticos son cada vez más frecuentes en la práctica dermatológica, presentando un importante aumento en las últimas décadas. El desarrollo de nuevas técnicas y métodos mínimamente invasivos han permitido obtener resultados estéticos satisfactorios con un menor tiempo de recuperación. No obstante, estos no se encuentran exentos de complicaciones, las que pueden ser transitorias o permanentes, por lo que su reconocimiento precoz y manejo adecuado permiten minimizar las secuelas. El objetivo de este artículo es revisar las principales complicaciones de los procedimientos dermatológicos más utilizados.
${ }^{1}$ Servicio de Dermatología, Clínica Las Condes, Santiago, Chile. ${ }^{2}$ Servicio de Dermatología, Hospital San José, Santiago, Chile.

Correspondencia: Patricia Apt Correo electrónico: papt@clc.cl Teléfono: +56 000000000 Dirección: Fray Montalva 712, Santiago, Chile.. Código Postal: 7590973.

\section{RELLENOS}

Los materiales de relleno están destinados al aumento de tejidos blandos, restaurando el volumen facial, permitiendo la corrección de arrugas y/o pliegues. ${ }^{1}$ Existe una amplia variedad de rellenos, clasificándose según su duración en biodegradables o reabsorbibles, donde encontramos al ácido hialurónico $(\mathrm{AH})$, colágeno, ácido poliláctico (APL), hidroxiapatita de calcio (HaCa) y grasa autóloga, y el grupo de los no biodegradables o permanentes, compuesto por la silicona, metilmetacrilato y poliacrilamida. ${ }^{2} \mathrm{El} \mathrm{AH}$ es el único relleno que se puede disolver de forma enzimática, de manera rápida y segura mediante hialuronidasa, ${ }^{3}$ lo que otorga una ventaja significativa sobre los otros rellenos. 
Las complicaciones más frecuentes son leves y transitorias, generalmente relacionadas con la aplicación del relleno, mientras que las complicaciones de mayor relevancia son infrecuentes, pudiendo ser temporales o permanentes. ${ }^{2}$

Equimosis: Es la complicación más frecuente asociada a la aplicación del relleno, ${ }^{3}$ especialmente en pacientes que consumen antiagregantes plaquetarios, AINES, anticoagulantes, vitamina E, Ginkgo Biloba, Ginseng o alcohol. Para su prevención se recomienda utilizar cánulas romas o agujas del menor grosor posible $(27 \mathrm{G}$ - 28G), administrar el relleno en forma lenta, en pequeñas cantidades, aplicar hielo antes y después de la administración y evitar fármacos que afecten la coagulación una a dos semanas previas. ${ }^{3,4}$ La mayoría se resuelve espontáneamente, el uso de árnica en forma tópica puede ayudar a acortar el tiempo de resolución. ${ }^{3}$

Edema: Es esperable que se genere después de la aplicación de cualquier relleno, ${ }^{5}$ especialmente en la región periorbitaria y en zonas altamente vascularizadas como los labios. ${ }^{3}$ Generalmente el edema es transitorio, resolviéndose en una a dos semanas. Se debe sospechar obstrucción linfática en caso de persistencia de edema en la región periorbitaria, ${ }^{5}$ la que no está asociada con eritema ni prurito. ${ }^{4}$ En esta región, el edema se puede deber a la aplicación del relleno superficial al septo malar o por aplicaciones de grandes volúmenes, generando una mayor presión en los vasos linfáticos. ${ }^{1}$ En su tratamiento se puede considerar el uso de corticoides orales y en caso de aplicación de $\mathrm{AH}$, usar hialuronidasa en forma gradual en intervalos semanales. ${ }^{6}$ Para su prevención se recomienda administrar escasa cantidad de relleno y situarlo a nivel del supraperiostio. ${ }^{1}$

Agregación del relleno: Se pueden percibir como nódulos asintomáticos especialmente en los párpados inferiores, ${ }^{7}$ posterior al uso de $\mathrm{AH}$ y $\mathrm{HaCa}$, los que se resuelven dentro de una a dos semanas y dentro de dos a seis semanas, respectivamente. ${ }^{5}$ En caso de persistencia con $\mathrm{AH}$, se puede utilizar hialuronidasa $10 \mathrm{a}$ 20 unidades por cada $0,1 \mathrm{ml}$ aplicado de AH 5 y en el caso de HaCa, se puede inyectar solución salina para diluirlo y hacerlo maleable, permitiendo su redistribución mediante masaje. ${ }^{5}$ En caso de disconfort cosmético o dolor, se puede aplicar acetónido de triamcinolona o 5-fluororacilo intralesional y/o excisión local. ${ }^{5}$

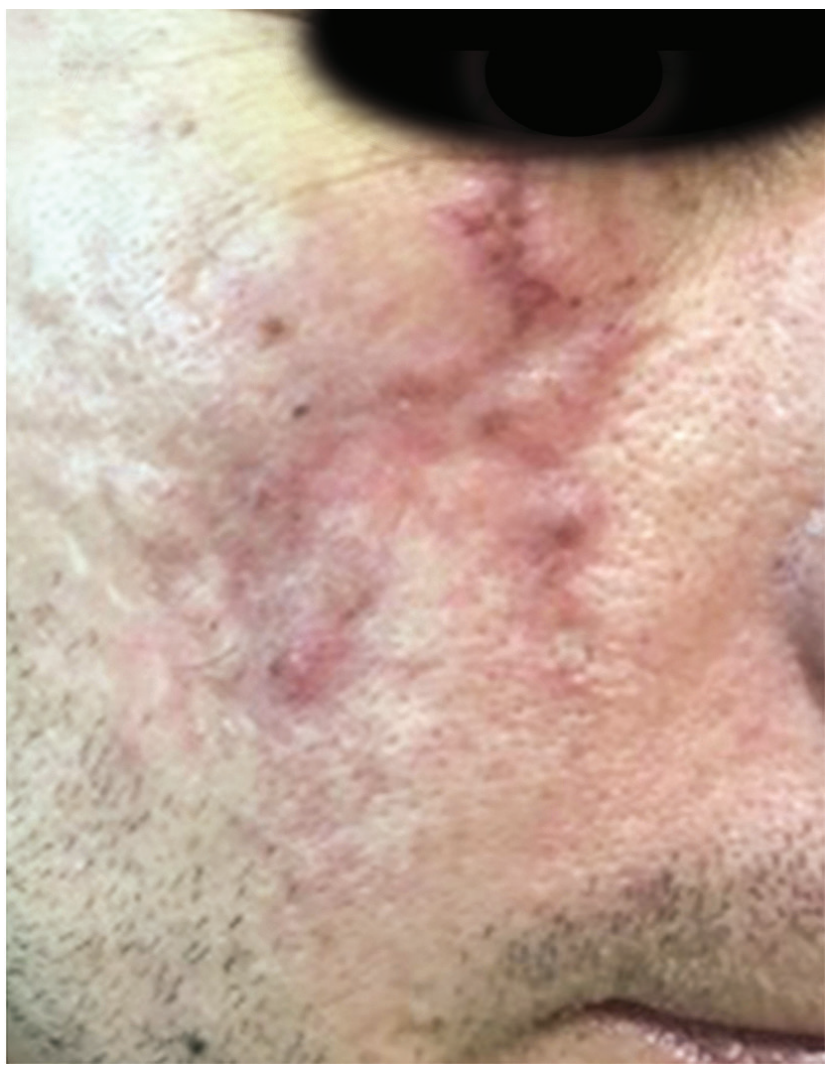

\section{Figura 1}

Infección bacteriana posterior a uso de relleno de cicatrices de acné.

Infección: Es una complicación infrecuente, que se debe sospechar frente a la presencia de edema o nódulo eritematoso doloroso dentro de los primeros días de la aplicación (FIgura 1). ${ }^{5}$ En las infecciones precoces el S. aureus es el patógeno más frecuente. ${ }^{3}$ En caso de persistencia por más de dos semanas, se deben descartar mycobacterias atípicas o biofilms, ${ }^{8}$ que corresponden a colecciones de microorganismos adheridos a la superficie del relleno, los que son resistentes al tratamiento antibiótico. ${ }^{1}$ En su prevención es esencial el uso de antisépticos y una técnica aséptica. ${ }^{3}$ En los pacientes con abscesos o nódulos persistentes, pueden realizase drenajes, corticoides intralesionales y/o extirpación quirúrgica. ${ }^{3}$

Efecto Tyndall: Los rellenos deben ser infiltrados en niveles específicos en la piel según su composición. El AH de alta densidad, la HaCa y el APL generan una coloración azul-grisácea en la piel cuando son depositados en forma superficial, debido al efecto Tyndall, siendo de mayor riesgo las áreas de piel delgada.3,5 
El APL se debe situar a nivel subcutáneo o supraperiosteo, la HaCa en la unión dérmica/subcutánea y el AH dependiendo de su densidad, a nivel subdérmico, subcutáneo o supraperiosteal. ${ }^{9}$ Se puede manejar con hialuronidasa en caso de uso de AH.

Hipersensibilidad tardía: Es infrecuente, estimándose en un $0,02 \%$ para $\mathrm{AH}^{10}$, con un menor riesgo para HaCa y APL. Se pueden desarrollar en forma tardía, meses a años después de la aplicación del relleno, debido a impurezas de los agentes químicos utilizados, por sustancias que aumentan la duración del relleno o por bioproductos residuales de la fermentación bacteriana. ${ }^{5}$ Las partículas de mayor tamaño del relleno son resistentes a la fagocitosis de los polimorfonucleares, requiriendo de la acción de los macrófagos, generándose granulomas. ${ }^{11}$ Su tratamiento es difícil y depende del tipo de relleno. Para rellenos de AH se puede utilizar hialuronidasa, mientras que para otros tipos de rellenos se puede indicar el uso de corticoides intralesionales o 5-fluororacilo, los que son inefectivos frente a HaCa. ${ }^{12}$ En caso de no respuesta, se puede realizar extirpación quirúrgica.

Complicaciones vasculares: Se producen de manera infrecuente, por compresión extrínseca o por su aplicación intravascular. ${ }^{2}$ Clínicamente se observa blanqueamiento cutáneo súbito y dolor. Las zonas de mayor riesgo son la glabela y el pliegue nasolabial. Se debe detener de forma inmediata su administración, masajear el área, administrar aspirina, compresas calientes y nitroglicerina al 2\% en ungüento. En caso de no respuesta, se indica la administración de hialuronidasa. ${ }^{5}$ Las dosis varían según distintas recomendaciones de expertos, utilizándose dosis iniciales de 200-300 unidades llegando hasta más de 1000 unidades para lograr reperfusión. ${ }^{13}$ En caso de no pesquisa a las 24 horas, se observará una coloración violácea moteada similar a una equimosis pero con un dolor intenso, pudiendo evolucionar a necrosis cutánea con posterior formación de cicatrices. ${ }^{5}$

La amaurosis súbita es la complicación más severa e infrecuente, debido a la embolización secundaria a la administración intravascular. Se produce principalmente por el uso de grasa autóloga, siendo generalmente permanente. ${ }^{5}$ Los sitios de mayor riesgo son la región glabelar, pliegue nasolabial y región temporal. Se previene administrando pequeñas cantidades del relleno, a baja velocidad, con cánulas romas y usando vasoconstrictor. En caso de sospecha, se debe derivar en forma urgente al oftalmólogo y administrar hialuronidasa retrobular en caso de uso de $\mathrm{AH} .{ }^{5,14}$

Alteración de tejidos: Se puede observar migración del relleno meses a años después de su aplicación, en los tejidos circundantes o distantes al sitio de administración original, generalmente con rellenos no biodegradables. ${ }^{5}$ Por esta razón, se contraindica el uso de $\mathrm{HaCa}$ en labios. Se puede utilizar hialuronidasa en caso de AH 5 o excisión quirúrgica en caso de otros materiales. ${ }^{5}$

\section{Peeling, Láser y LuZ Pulsada Intensa}

Los peeling generan una injuria controlada que varía en su profundidad dependiendo del producto, tiempo de aplicación y concentración utilizada, promoviendo la formación de colágeno, reduciendo el fotodaño y mejorando la textura de la piel. ${ }^{15}$ Según la profundidad del daño se clasifican en; muy superficiales, superficiales, medios y profundos, alcanzando el estrato córneo, estrato basal de epidermis, dermis superficial y dermis reticular, respectivamente. ${ }^{1}$

Las terapias de luz son ampliamente utilizadas por dermatólogos, indicándose para rejuvenecimiento cutáneo, depilación, remoción de tatuajes y lesiones vasculares. Los láser ablativos tienen buenos resultados estéticos pero con mayor riesgo de complicaciones y mayor periodo de recuperación. ${ }^{16}$ Los nuevos láser ablativos fraccionados generan una fototermolisis parcial del área a tratar, lo que ha permitido disminuir las complicaciones y el tiempo de recuperación. ${ }^{1}$ A su vez, los procedimientos no ablativos como la luz pulsada intensa, láser vasculares, láser infrarrojos, ultrasonido y radiofrecuencia, mantienen la epidermis intacta, con menor riesgo de complicaciones, pero con menor eficacia clínica respecto a los láser ablativos. ${ }^{17}$

Los eventos adversos de los peeling y láser ablativos son similares debido al daño epidérmico y dérmico que producen. Entre las principales complicaciones se encuentran el eritema, edema, equimosis, aumento de la sensibilidad cutánea, prurito, dolor, descamación, discromías e infecciones. ${ }^{1}$ Otras complicaciones menos frecuentes son las cicatrices, eritema prolongado, 
milia/acné, reacciones alérgicas y toxicidad sistémica (nefrológica, hepática y/o cardíaca), este último en peeling con fenol, resorcinol y/o ácido salicílico. ${ }^{18}$

Eritema, edema y equimosis: La reacción inflamatoria aguda de la piel es esperable, la que se resuelve entre dos a cuatro días en los procedimientos no ablativos y entre dos a cuatro semanas en los láser ablativos. ${ }^{3}$ Los pacientes con rosácea, fototipos bajos y eritrosis facial son más propensos al eritema. Se considera como eritema prolongado, la persistencia por más de cuatro días en los láser no ablativos y por más de un mes en láser ablativos, presentándose en el 1\% y $12,5 \%$, respectivamente (Figura 2 ) ${ }^{17}$ Estas reacciones se pueden minimizar utilizando hielo en forma precoz y humectación frecuente. La equimosis se produce posterior al uso de láser decolorante pulsado, luz pulsada intensa, láser Nd:YAG o KTP. Se resuelve en forma espontánea y se deben evitar fármacos que alteren la coagulación. ${ }^{15}$

Prurito: Habitualmente comienza entre dos a tres días después del procedimiento, siendo normal hasta un mes posterior al peeling. ${ }^{15} \mathrm{El}$ prurito intenso es anormal, pudiendo ser un signo de infección por virus herpes simplex, cándida o una dermatitis de contacto alérgica. ${ }^{15}$

Costras: Luego de un láser ablativo o de un peeling profundo, es esperable la presencia de costras asintomáticas. Si estás persisten por más de tres días, se pueden indicar pulsos breves de corticoides tópicos de alta potencia o hidrocoloides, asociados a apósitos que retengan humedad. ${ }^{19}$ Se debe sospechar infección en pacientes con costras prolongadas, mal olor y ulceración.

Infecciones: Son causadas por virus, bacterias y hongos, las que generalmente se presentan durante la primera semana. ${ }^{17}$ Sin embargo, las infecciones por micobacterias atípicas se pueden reportar hasta 2 meses después. ${ }^{20}$ La reactivación del virus herpes simplex es la infección más frecuente, la que se produce en un $0,5 \%$ a $5 \%$ de los procedimientos ablativos o peeling medios/profundos. ${ }^{21}$ Para su profilaxis, se indica valaciclovir un día previo al procedimiento hasta la reepitelización completa, disminuyendo el riesgo de reactivación herpética. Clínicamente se presenta con prurito, dolor, exudado y erosiones superficiales, sin presentarse con las clásicas vesículas de infección

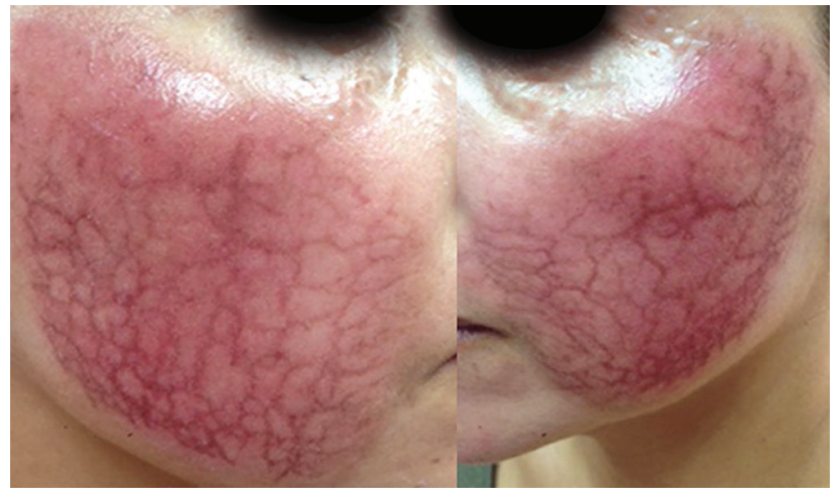

Figura 2

Eritema persistente posterior a peeling.

herpética. ${ }^{22}$ La infección por hongos más frecuente es por Candida albicans, manifestándose a los 7 a 14 días post procedimiento con prurito intenso. ${ }^{17}$

Erupción acneiforme y milia: Las reacciones acneiformes se producen dentro de uno a cinco días después de los procedimientos donde se genera disrupción de la unidad folicular, más frecuente en los peeling medios/profundos y Co2 fraccionado, observándose en el 5 a $10 \%$ de los $\operatorname{casos}^{3}{ }^{3}$ La milia se presenta dos a tres semanas después del procedimiento, en el $19 \%$ de los casos, 16 lo que se exacerba frente al uso de cremas oclusivas. En el tratamiento de las erupciones acneiformes leves a moderadas se indican antibióticos tópicos u orales, y en casos severos se utilizan corticoides orales o intralesionales. ${ }^{15} \mathrm{El}$ tratamiento de la milia es mediante extracción manual, retinoides tópicos o dermoabrasión. ${ }^{3,15}$

Discromía: La hiperpigmentación postinflamatoria es una de las complicaciones más frecuentes, presentándose varias semanas posterior al procedimiento, siendo transitoria en la mayoría de los casos pero pudiendo persistir por varios meses. ${ }^{3,21}$ Los pacientes con mayor riesgo son los fototipos III o mayores, con exposición solar previa o posterior al procedimiento, ${ }^{23}$ discromías previas, medicamentos fotosensibilizadores, fotodaño severo y tratamiento láser con alta energía. ${ }^{15}$ Para su prevención se debe indicar fotoprotección estricta dos a cuatro semanas antes y dos a cuatro meses posterior al procedimiento. ${ }^{3}$ La hidroquinona, ácido kójico, retinoides tópicos u otros despigmentantes son útiles para la prevención en pacientes de alto riesgo y para su tratamiento. 


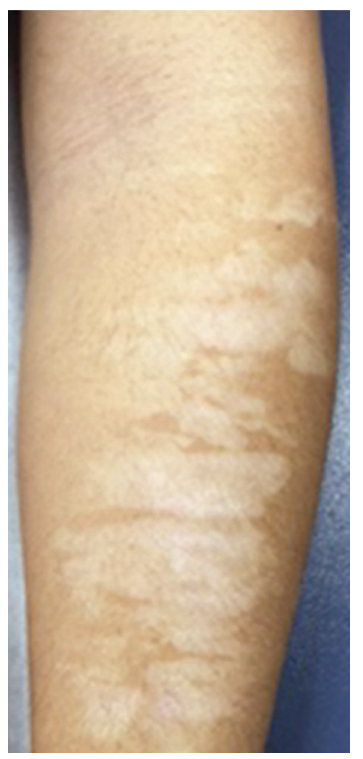

Figura 3

Hipopigmentación posterior a depilación por luz pulsada.

En su contraparte, la hipopigmentación es infrecuente y generalmente permanente, mayormente asociado al Láser Co2 y peeling profundos, siendo infrecuente en láser fraccionados (Figura 3). Entre los factores de riesgo se encuentran los pacientes con fototipos altos y antecedentes de peeling profundos.3 Puede existir repigmentación espontánea que se produce en tres a cuatros meses, en caso contrario se puede utilizar láser excimer o camuflaje. ${ }^{24}$

Cicatrices: Son infrecuentes, causadas por un daño termal excesivo, incapacidad regenerativa o secundaria a procesos infecciosos. ${ }^{3}$ Los factores de riesgo son antecedentes de queloides, tabaquismo, patologías crónicas, infecciones y cuidados inadecuados posteriores al procedimiento. Las cicatrices excesivas se pueden manejar con láminas de siliconas, corticoides o 5 fluororacilo intralesional. Frente a cicatrices con eritema excesivo se puede utilizar láser decolorante pulsado o luz pulsada intensa y, en caso de cicatrices atróficas, láser ablativos fraccionados. ${ }^{25}$

\section{ULTRASONIDO DE ALTA FREGUENGIA}

Genera un aumento de la temperatura del tejido subcutáneo, produciendo coagulación térmica selectiva sin comprometer la epidermis y dermis papilar, estimulando la formación de colágeno, mejorando la tensión y reposicionando los tejidos, generando un efecto lifting a nivel facial o extrafacial. ${ }^{26,27}$ Es un método no

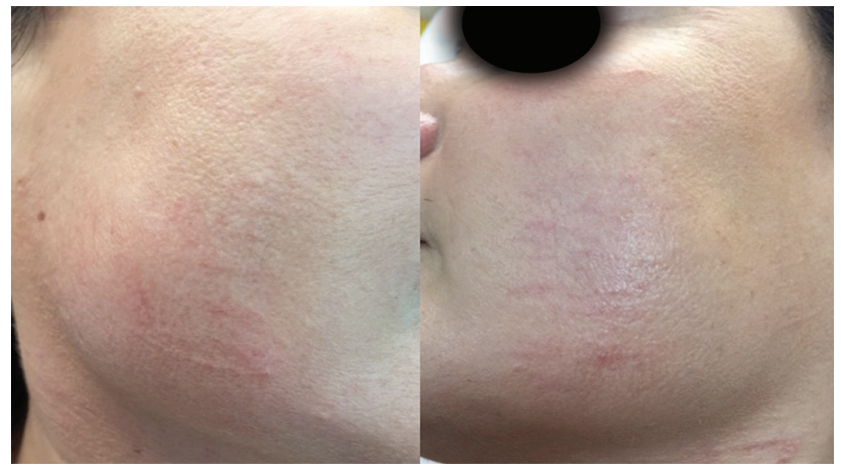

Figura 4

Placas eritematosas estriadas por uso de transductor de ultrasonido.

invasivo con complicaciones leves y transitorias; eritema que se resuelve antes de veinticuatro horas, edema leve entre dos a cuatro semanas, disestesias entre dos a cuatro semanas y equimosis en forma ocasional.26 Otra complicación que ocurre en caso de utilización de transductores superficiales con excesivo uso de gel, es el desarrollo de placas eritematosas estriadas, debido a que se genera coagulación térmica a nivel más superficial de la piel (Figura 4). También se ha descrito el compromiso transitorio de ramas superficiales del nervio facial en el $0,005 \%$ de los casos, generando asimetrías transitorias, con recuperación de la funcionalidad en dos a cuatro semanas. ${ }^{26}$

\section{TATUajes}

Son partículas de pigmentos exógenos insolubles que se depositan en la dermis, pueden ser utilizados como arte corporal, cosmético o terapéutico para mejorar apariencia de párpados, cejas, contorno de labios o cicatrices. ${ }^{28}$ Las partículas de pigmento depositadas en la dermis presentan una mínima tasa de eliminación mediante vía linfática llegando a los linfonodos regionales. ${ }^{29}$ Las principales complicaciones son las infecciosas, debido a las múltiples infiltraciones de la aguja en la dermis, siendo los principales microorganismos involucrados el Staphylococcus, Streptococcus, Pseudomonas, Escherichia coli y Clostridium spp. y de forma menos frecuente por micobacterias. Las infecciones se pueden manifestar como impétigo, abscesos, erisipela o celulitis, requiriendo tratamiento con antibióticos sistémicos. ${ }^{29}$ 


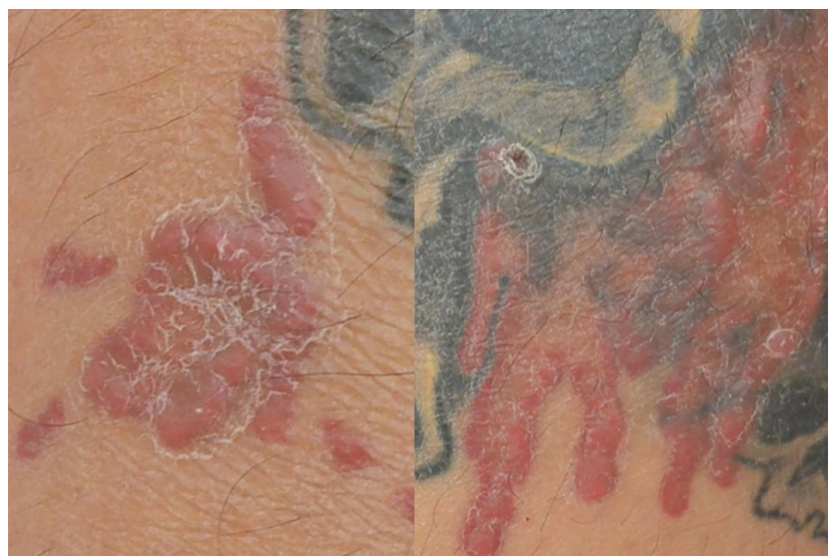

Figura 5

Reacción alérgica al pigmento rojo por tatuaje.

Dentro de las complicaciones no infecciosas, destacan las reacciones alérgicas, que se generan frente a un color específico en los sitios infiltrados, con una latencia de aparición de semanas, meses u años, siendo permanente y refractaria a los corticoides tópicos, 29 con estudio de test de parche negativo frente a estos alérgenos ${ }^{30}$. Las reacciones alérgicas se observan principalmente con el color rojo (Figura 5) y son menos frecuentes con el verde o azul. La manifestación principal es mediante una placa de superficie plana, bien delimitada, descamativa, en los lugares donde se utilizó el pigmento determinado.29 Cuando estas lesiones aumentan su grosor se denominan placas hiperqueratósicas. Una variante más severa son lesiones úlcero-necróticas. ${ }^{29}$

De igual forma se pueden presentar manifestaciones no inmunomediadas, secundarias a la infiltración excesiva de pigmento, generalmente frente al color negro, donde se puede observar la migración del pigmento mediante vía linfática o una reacción pápulo-nodular, que no compromete toda la región donde se aplicó el mismo pigmento, resolviéndose en forma espontánea al disminuir la cantidad de pigmento con el tiempo. Se pueden producir cicatrices hipertróficas o queloides secundarias al trauma múltiple de las agujas. ${ }^{29}$

\section{Conclusiones}

Los procedimientos estéticos son cada vez más relevantes para el dermatólogo, tanto como por su indicación, realización y por el manejo de las complicaciones. Es de vital importancia conocer, diagnosticar y manejar las complicaciones de los diversos procedimientos estéticos dermatológicos.

\section{REFERENCIAS}

1. Hartmann D, Ruzicka T, Gauglitz GG. Complications associated with cutaneous aesthetic procedures. J Dtsch Dermatol Ges. 2015;13:778-86

2. Kulichova D, Borovaya A, Ruzicka T, Thomas P, Gauglitz GG. Understanding the safety and tolerability of facial filling therapeutics. Expert Opin Drug Saf. 2014;13:1215-26

3. Hassouneh B, Newman JP. Lasers, fillers, and neurotoxins: avoiding complications in the cosmetic facial practice. Facial Plast Surg Clin North Am. 2013;21:585-98

4. Funt DK. Avoiding malar edema during midface/cheek augmentation with dermal fillers. J Clin Aesthet Dermatol. 2011;4:32-6

5. Vanaman M, Fabi SG, Carruthers J. Complications in the Cosmetic Dermatology Patient: A Review and Our Experience (Part 1). Dermatol Surg. 2016;42:1-11

6. Hilton S, Schrumpf H, Buhren BA, Bolke E, Gerber PA. Hyaluronidase injection for the treatment of eyelid edema: a retrospective analysis of 20 patients. EurJ Med Res. 2014;19:30

7. Daines SM, Williams EF. Complications associated with injectable soft-tissue fillers: a 5-year retrospective review. JAMA Facial Plast Surg. 2013;15:226-31

8. Smith KC. Reversible vs. nonreversible fillers in facial aesthetics: concerns and considerations. Dermatol Online J. 2008;14:3

9. Carruthers J, Rzany B, Sattler G, Carruthers A. Anatomic guidelines for augmentation of the cheek and infraorbital hollow. Dermatol Surg. 2012; 38(7 Pt 2):1223-33

10. Friedman PM, Mafong EA, Kauvar AN, Geronemus RG. Safety data of injectable nonanimal stabilized hyaluronic acid gel for soft tissue augmentation. Dermatol Surg. 2002;28:491-4

11. O'Reilly P, Malhotra R. Delayed hypersensitivity reaction to Restylane (R) SubQ. Orbit. 2011;30:54-7

12. Lemperle G, Rullan PP, Gauthier-Hazan N. Avoiding and treating dermal filler complications. Plast Reconstr Surg. 2006;118(3 Suppl):92s-107s

13. Cohen JL, Biesman BS, Dayan SH, DeLorenzi C, Lambros VS, Nestor MS, et al. Treatment of Hyaluronic Acid FillerInduced Impending Necrosis With Hyaluronidase: Consensus Recommendations. Aesthet Surg J. 2015;35:844-9

14. Kim DW, Yoon ES, Ji YH, Park SH, Lee BI, Dhong ES. Vascular complications of hyaluronic acid fillers and the role of hyaluronidase in management. J Plast Reconstr Aesthet Surg. 2011;64:1590-5

15. Vanaman M, Fabi SG, Carruthers J. Complications in the Cosmetic Dermatology Patient: A Review and Our Experience (Part 2). Dermatol Surg. 2016;42:12-20

16. Alster TS, Tanzi EL. Complications in laser and light surgery. In: Golberg DJ, editor. Lasers and Lights, Volume 2. Philadelphia, PA: Saunders Elsevier; 2008. p. 99-112

17. Metelitsa AI, Alster TS. Fractionated laser skin resurfacing treatment complications: a review. Dermatol Surg. 2010;36:299-306

18. Khunger N. Standard guidelines of care for chemical peels. Indian J Dermatol Venereol Leprol. 2008;74 Suppl:S5-12

19. Alam M, Warycha M. Complications of lasers and light treatments. Dermatol Ther. 2011;24:571-80 
20. Palm MD, Butterwick KJ, Goldman MP. Mycobacterium chelonae infection after fractionated carbon dioxide facial resurfacing (presenting as an atypical acneiform eruption): case report and literature review. Dermatol Surg. 2010;36:1473-81

21. Graber EM, Tanzi EL, Alster TS. Side effects and complications of fractional laser photothermolysis: experience with 961 treatments. Dermatol Surg. 2008;34:301-5; discussion 5-7

22. Setyadi HG, Jacobs AA, Markus RF. Infectious complications after nonablative fractional resurfacing treatment. Dermatol Surg. 2008;34:1595-8

23. Handley JM. Adverse events associated with nonablative cutaneous visible and infrared laser treatment. J Am Acad Dermatol. 2006;55:482-9

24. Alexiades-Armenakas MR, Bernstein LJ, Friedman PM, Geronemus RG. The safety and efficacy of the 308-nm excimer laser for pigment correction of hypopigmented scars and striae alba. Arch Dermatol. 2004:140:955-60

25. Alster TS, Tanzi EL, Lazarus M. The use of fractional laser photothermolysis for the treatment of atrophic scars. Dermatol Surg. 2007;33:295-9

26. Fabi SG. Microfocused ultrasound with visualization for skin tightening and lifting: my experience and a review of the literature. Dermatol Surg. 2014;40 Suppl 12:S164-7

27. Fabi SG, Massaki A, Eimpunth S, Pogoda J, Goldman MP. Evaluation of microfocused ultrasound with visualization for lifting, tightening, and wrinkle reduction of the decolletage. J Am Acad Dermatol. 2013;69:965-71

28. Vassileva S, Hristakieva E. Medical applications of tattooing. Clin Dermatol. 2007;25:367-74

29. Serup J, Carlsen KH, Sepehri M. Tattoo complaints and complications: diagnosis and clinical spectrum. Curr Probl Dermatol. 2015;48:48-60

30. Serup J, Hutton Carlsen K: Patch test study of 90 patients with tattoo reactions: negative outcome of allergy patch to baseline batteries and culprit inks suggests allergen $(\mathrm{s})$ are generated in the skin through haptinization. Contact Dermatitis. 2014;71:255-263 